\title{
Designs of fullerene-based frameworks for hydrogen storage
}

Cite this: J. Mater. Chem. A, 2014, 2 , 5910

Received 28th August 2013 Accepted 17th January 2014

DOI: 10.1039/c3ta13426a

www.rsc.org/MaterialsA

\begin{abstract}
Yi Gao, ${ }^{\text {*a }}$ Xiaojun Wu ${ }^{b}$ and Xiao Cheng Zeng ${ }^{c b}$
Two types of hybrid metallofullerene framework are theoretically designed, and their structural stabilities are examined using the density functional theory (DFT) computation. Both frameworks are constructed by connecting exohedral metallofullerene nodes with conjugated organic linkers, akin to the common metal-organic framework (MOF). The DFT calculations suggest that hydrogen molecules can be adsorbed in the frameworks with the hydrogen binding energies ranging from $0.15-0.50 \mathrm{eV}$, satisfying the optimal adsorption condition for hydrogen storage. Moreover, our computation suggests that the frameworks can entail molecular $\mathrm{H}_{2}$ binding in the range of 8.0-9.2 wt\%, meeting the Department of Energy (DOE) target of 2010 or 2015.
\end{abstract}

\section{Introduction}

Hydrogen holds the promise as one of the major energy resources in place of the fossil fuel. ${ }^{1}$ For future automobile applications, the US DOE has set the $6.0 \mathrm{wt} \%$ and $9.0 \mathrm{wt} \%$ onboard hydrogen storage capacity as targets for the year of 2010 and 2015. ${ }^{2}$ To date, a material with high gravimetric/volumetric density of hydrogen molecules while being able to operate reversibly under moderate temperature and pressure conditions is still lacking. It is known that storage of hydrogen molecules in either gas or liquid state is undesirable for automobile applications due to either too low volumetric density or the prerequisite of high-energy cost for hydrogen liquefaction. Hence, seeking ideal solid-state hydrogen-storage materials has been a central focus of recent research. Today, metal/complex hydrides and nano-structured materials have been widely viewed as two most promising solid materials for hydrogen storage. $^{3-14}$ High gravimetric density $(>10 \mathrm{wt} \%)$ can be easily achieved for solids containing only light elements such as $\mathrm{Li}$, $\mathrm{Be}, \mathrm{B}, \mathrm{C}, \mathrm{Na}, \mathrm{Mg}$, and $\mathrm{Al}$. One subtlety of utilizing metal/complex hydrides is that hydrogen is stored either in the form of strong covalent bond or ionic bond, while a subtlety of using nanostructured materials is that hydrogen is stored in the form of weakly van der Waals (vdW) bond. Thus the stored hydrogen would be either very difficult to desorb or too easy to desorb at low temperature. Although notable progress has been made

${ }^{a}$ Division of Interfacial Water and Key Laboratory of Interfacial Physics and Technology, Shanghai Institute of Applied Physics, Chinese Academy of Sciences, Shanghai, 201800 China. E-mail: gaoyi@sinap.ac.cn; Tel: +86 18821256250

${ }^{b}$ Department of Materials Science and Engineering, University of Science and Technology of China, Hefei, 230026 China. E-mail: xjwu@ustc.edu.cn; Tel: +86 13866730423

'Department of Chemistry, University of Nebraska-Lincoln, Lincoln, Nebraska, 68588 USA.E-mail: xzeng1@unl.edu; Tel: +1 4024729894 towards meeting the DOE's targets for hydrogen storage, the ideal storage system is yet to be synthesized.

The optimal $\mathrm{H}_{2}$ adsorption energy is in the range of 0.2-0.6 $\mathrm{eV}$ per $\mathrm{H}_{2}$ for retrievable storage and kinetic requirement in ambient condition. ${ }^{15,16}$ Some early experiments have shown that pristine carbon nanotubes can store hydrogen but with only limited capacity. ${ }^{17-19}$ Further experiments shown the addition of transition metal could enhance binding energies for nondissociative hydrogen adsorption on single-walled $(\sim 20 \mathrm{~kJ}$ $\left.\mathrm{mol}^{-1}\right)$ and multi-walled carbon nanotubes $\left(\sim 54 \mathrm{~kJ} \mathrm{~mol}^{-1}\right) \cdot{ }^{20,21}$ The hydrogen binding energies are significantly stronger than vdW interaction between $\mathrm{H}_{2}$ and the carbon nanotubes, but much less than the chemisorption energy of the atomic hydrogen. Recently, some theoretical studies predicted a number of organometallic carbon fullerenes and nanotubes as potential hydrogen storage materials. Sun et al. employed DFT and investigated the interaction between the hydrogen and Ticoated fullerenes. They showed that Ti tends to form metal clusters on the fullerene surface rather than to cover the surface uniformly. Such a clustering tendency prevents Ti being an effective covering element on fullerenes for binding hydrogen molecules. ${ }^{22}$ Later, Sun predicted that a buckyball $\mathrm{C}_{60}$ can be coated with twelve $\mathrm{Li}$ atoms on which total $60 \mathrm{H}_{2}$ can be adsorbed with the adsorption energy of $0.075 \mathrm{eV}$ per $\mathrm{H}_{2}{ }^{23}$ More importantly, they showed that the uniformly Li-coated fullerene is energetically more favorable than the fullerene combined with a Li-cluster. Zhang and co-workers investigated hydrogen storage capability of TM-coated $\mathrm{C}_{60}$ and $\mathrm{C}_{48} \mathrm{~B}_{12}$ fullerenes. They predicted that the maximum reversible hydrogen storage in these systems can be as high as $9 \mathrm{wt} \%{ }^{21} \mathrm{Wu}$ et al. suggested that Li-coated boron carbide nanotubes could store $\mathrm{H}_{2}$ with more than $6 \mathrm{wt} \%$ and with the binding energy in the range of $10-24 \mathrm{~kJ}$ $\mathrm{mol}^{-1},{ }^{24 a}$ and so could the edge-modified graphene nanoribbons with $\mathrm{Sc}^{24 b}$ Sun et al. also suggested that Li-coated and 
B-doped heterofullerene $\left(\mathrm{Li}_{12} \mathrm{C}_{48} \mathrm{~B}_{12}\right)$ could store hydrogen up to $9 \mathrm{wt} \% .^{25 a}$ Some recent theoretical studies by Yoon et al. ${ }^{25 b}$ and Wang et al. ${ }^{26}$ suggested that the Ca-coated fullerenes can reach the hydrogen uptake of $8.4 \mathrm{wt} \%$, close to the DOE 2015 target.

We also note that metal-organic framework (MOF) systems have attracted much attention due to their relatively high hydrogen uptake at low temperature. ${ }^{\mathbf{1 0 , 1 1}}$ For example, isoreticular metal-organic framework-1 (IRMOF-1) can store $1.3 \mathrm{wt} \%$ hydrogen, and isoreticular metal-organic framework11 (IRMOF-11) can store $1.6 \mathrm{wt} \%$ hydrogen at $77 \mathrm{K.}{ }^{27}$ At room temperature and pressure of 10 bar, hydrogen uptake of $2 \mathrm{wt} \%$ has been detected for the isoreticular metal-organic framework8 (IRMOF-8). ${ }^{10}$ It has also been reported that metal-organic framwork-5 (MOF-5) can adsorb up to $4.5 \mathrm{wt} \%$ hydrogen at $78 \mathrm{~K}$ but only $1 \mathrm{wt} \%$ at the room temperature and 20 bar. Another experiment demonstrates that the adsorption of hydrogen in MOF-177 and IRMOF-20 saturates between 70 and 80 bar, within which the $\mathrm{H}_{2}$ uptake can be as high as 7.5 and $6.7 \mathrm{wt} \%$, respectively. ${ }^{28}$ Dincă et al. reported a novel MOF with exposed $\mathrm{Mn}^{2+}$ coordination sites and a previously unknown cubic topology. ${ }^{29}$ This MOF is capable of $\mathrm{H}_{2}$-uptake of $6.9 \mathrm{wt} \%$ at $77 \mathrm{~K}$ and 90 bar. Lastly, the covalent-organic frameworks synthesized in Yaghi group can provide more flexible options as hydrogen storage systems. ${ }^{30}$

Although MOF is a promising candidate for hydrogen storage, their adsorption capacities are yet to meet the DOE targets for practical applications. To improve the hydrogen binding capability, Yang et al. introduced Li-cations in the anionic MOF built from In(III) center and tetracarboxylic acid ligands, and they found enhanced $\mathrm{H}_{2}$ adsorption as well as an increase in the isosteric heat of adsorption. ${ }^{31}$ Yaghi and coworkers synthesized MOF materials with ultrahigh porosity ${ }^{32}$ and large-pore apertures ${ }^{33}$ to further increase the hydrogenstorage capacity. Meanwhile, Bacsa et al. designed several fullerene-based MOF materials. ${ }^{34}$ Very recently, Rao et al. suggested encapsulated buckyballs in the cavity of Li-doped MOF and predicted ultra-high $\mathrm{H}_{2}$ uptakes at room temperature. ${ }^{35}$ Han and Goddard also predicted that the Li-doped MOF can lead to a gravimetric $\mathrm{H}_{2}$ uptake of $6.0 \mathrm{wt} \%$ which just meets the 2010 DOE target. ${ }^{36}$

In this article, we present a new design strategy to combine advantages of both hydrogen-storage nanomaterials and MOF. Specifically, we propose two new frameworks, one threedimensional (FW-1) and one two-dimensional (FW-2), with the exohedral metallofullerenes as nodes and organic molecules as linkers. Based on DFT calculations, we find that both FW-1 and FW-2 could store $\mathrm{H}_{2}$ above $8.0 \mathrm{wt} \%$, which meets the 2010 DOE target.

\section{Computational methods}

The first framework, FW-1 (see Fig. 1), is composed of $\mathrm{Li}_{8} @ \mathrm{C}_{48} \mathrm{~B}_{12}$ nodes and Li-doped benzenedicarboxylate (BDC) linkers. The second framework, FW-2, is a $2 \mathrm{D}$ network which is built upon the $\mathrm{Ca}_{32} \mathrm{C}_{60}$-node and $p$-dihydroxybenzene linker (see Fig. 2). FW-1 and FW-2 possess cubic and hexagonal unit cells, respectively. For FW-2, the distance between sheets is set as $15 \AA$, which is large enough to neglect interactions among periodic images. All geometries are optimized and the lattice is relaxed using $\mathrm{LDA}-\mathrm{PWC}^{37}$ and GGA-PBE ${ }^{38}$ DFT method, respectively. Both LDA-PWC and GGA-PBE methods are also employed to compute the $\mathrm{H}_{2}$ binding energies on the MOF fragments systematically. The values obtained can be viewed as the lowerlimit and upper-limit of the $\mathrm{H}_{2}$ binding energies. ${ }^{39}$ Furthermore, Grimme dispersion correction $\left(D_{2}\right)$ is used to evaluate weak interactions, ${ }^{40}$ and to compare with LDA and PBE calculations. Semi-core pseudo-potential is used together with the double numerical plus polarization basis set (DNP). ${ }^{\mathbf{4 1}}$ The SCF convergence is set as $10^{-5}$ Hartree. The global orbital cutoff is $4.80 \AA$. The energy threshold for geometry optimization is $2 \times$ $10^{-5}$ Hartree. All calculations are carried out using the Dmol3 software package. ${ }^{41}$ The binding energies between $\mathrm{H}_{2}$ and frameworks are defined as $\Delta E_{\text {binding }} / \mathrm{H}_{2}=\left(\mathrm{N}_{\mathrm{H}_{2}} \times E\left(\mathrm{H}_{2}\right)+E(\mathrm{FW})\right.$ $\left.-E\left(\mathrm{~N}_{\mathrm{H}_{2}}-\mathrm{FW}\right)\right) / \mathrm{N}_{\mathrm{H}_{2}}$.

\section{Results and discussion}

$\mathrm{H}_{2}$ molecules are introduced one by one into the supercell of the framework $\mathrm{FW}-1$. The maximum number of $\mathrm{H}_{2}$ adsorbed on each $\mathrm{Li}$ and oxygen atom is three and two, respectively. It was suggested that $\mathrm{Li}^{+}$ion could bind up to six $\mathrm{H}_{2}$ molecules with the binding energies in the range of 0.202 to $0.253 \mathrm{eV}$ per $\mathrm{H}_{2}{ }^{42}$ However, the neutral $\mathrm{Li}$ atoms on fullerenes can adsorb up to three $\mathrm{H}_{2}$ molecules due to the lack of the positive charge. ${ }^{25 a}$ Hence, the maximum $\mathrm{H}_{2}$ loading per supercell is up to $66 \mathrm{H}_{2}$ molecules (see Fig. 3). The cell length and corresponding $\mathrm{H}_{2}$ binding energies of system 1 are collected in Table 1 . Based on the DFT-PWC calculations, the optimized unit-cell length (17.60 $\mathrm{\AA})$ at low hydrogen loadings is slightly longer than that $(17.55 \AA$ ) without the hydrogen adsorption. On the other hand, all DFT-PBE results give the same optimized unit-cell length $(17.80 \AA)$. The distances between an $\mathrm{H}_{2}$ molecule and Li atom are within $2.10 \AA$ based on LDA-PWC calculation, and $2.30 \AA$ based on GGA-PBE calculation. Similarly, the distance between $\mathrm{H}_{2}$ and the $\mathrm{O}$ atom are shorter than $2.80 \AA$ based on LDA-PWC

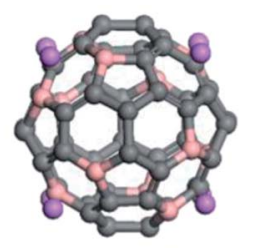

(a) Node: $\mathrm{Li}_{8} @ \mathrm{~B}_{12} \mathrm{C}_{48}$

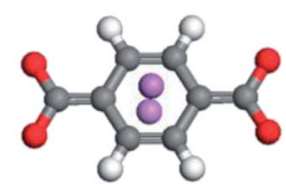

(b) Linker: Li-doped benzenecarboxylate

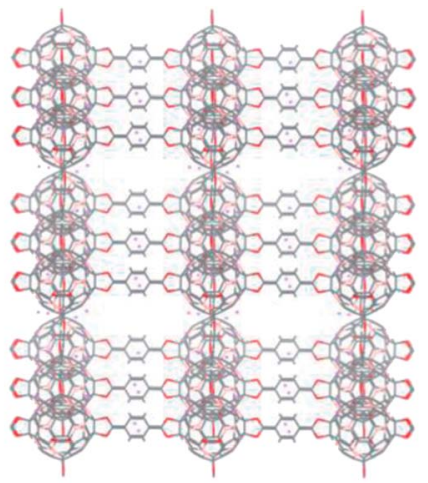

(c) FW-1: 3-D framework
Fig. 1 (a) Node: $\mathrm{Li}_{8}\left(\mathrm{aC}_{48} \mathrm{~B}_{12}\right.$. (b) Linker: Li-doped benzenedicarboxylate. (c) 3-D organic framework constructed by $\mathrm{Li}_{8} \mathrm{CC}_{48} \mathrm{~B}_{12}$ and $\mathrm{Li}-$ doped benzenedicarboxylate linker, names as FW-1. Red: oxygen; grey: carbon; white: hydrogen; purple: lithium. 


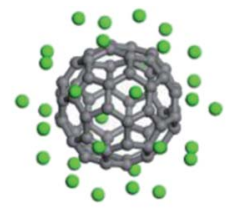

(a) Node: $\mathrm{Ca}_{32} \mathrm{C}_{60}$

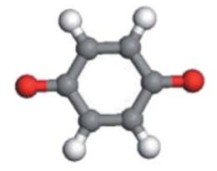

(b) Linker: $p$-dihydroxybenzene

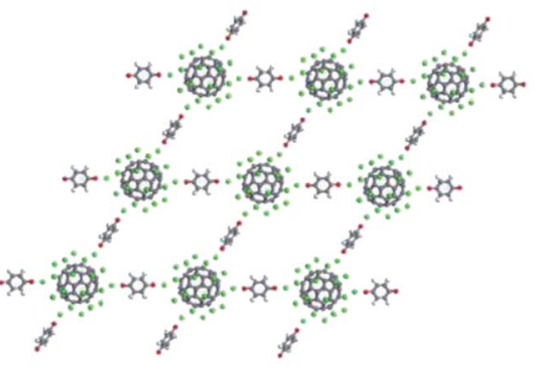

(c) FW-2: 2-D framework

Fig. 2 Optimized structure of (a) node: $\mathrm{Ca}_{32} \mathrm{C}_{60}$; (b) linker: $p$-dihydroxybenzene. (c) 2-D film constructed by $\mathrm{Ca}_{32} \mathrm{C}_{60}$ and $p$ dihydroxybenzene, named as FW-2. Red: oxygen; grey: carbon; white: hydrogen; green: calcium.

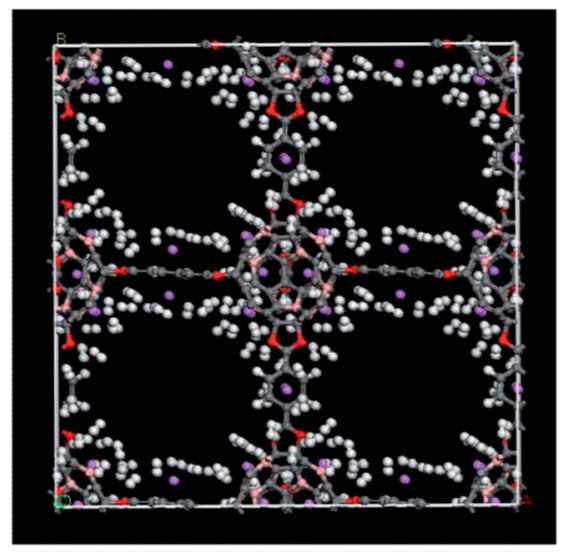

Fig. 3 Optimized structure of the Li-coated $\mathrm{C}_{48} \mathrm{~B}_{12}$ fullerene framework (FW-1) with $66 \mathrm{H}_{2}$ adsorbed within the supercell.

calculation, and 3.20 A based on GGA-PBE calculation. Meanwhile, the $\mathrm{H}-\mathrm{H}$ bond lengths are also elongated from $0.764 \AA$ to $0.780 \AA$ based on LDA-PWC calculations, and from $0.751 \AA$ to $0.755 \AA$ based on GGA-PBE calculations. These results indicate that hydrogen molecules interact with Li atoms or oxygen atoms via vdW interactions. The binding-energy ranges from $0.20 \mathrm{eV}$ per $\mathrm{H}_{2}$ to $0.26 \mathrm{eV}$ per $\mathrm{H}_{2}$ from DFT-PWC calculations, greater than that from DFT-PBE calculations $\left(0.07 \mathrm{eV}\right.$ per $\mathrm{H}_{2}$ to $0.14 \mathrm{eV}$

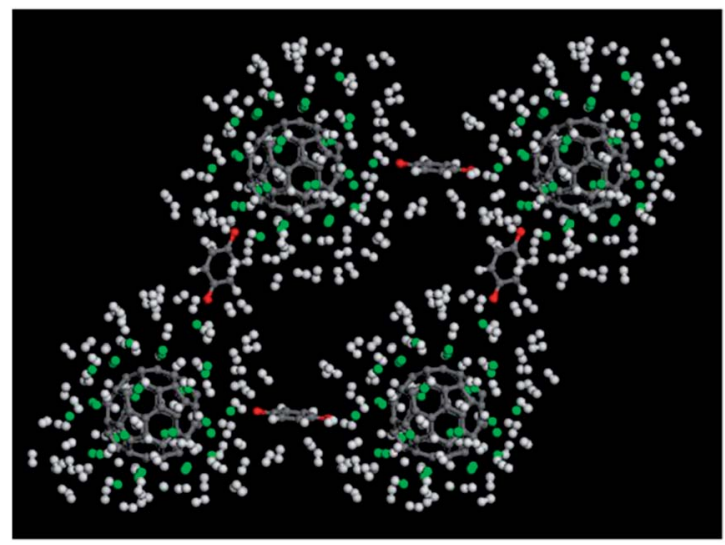

Fig. 4 Optimized structure of $96 \mathrm{H}_{2}-\mathrm{Ca}_{32} \mathrm{C}_{60} 2$-dimensional framework (FW-2).
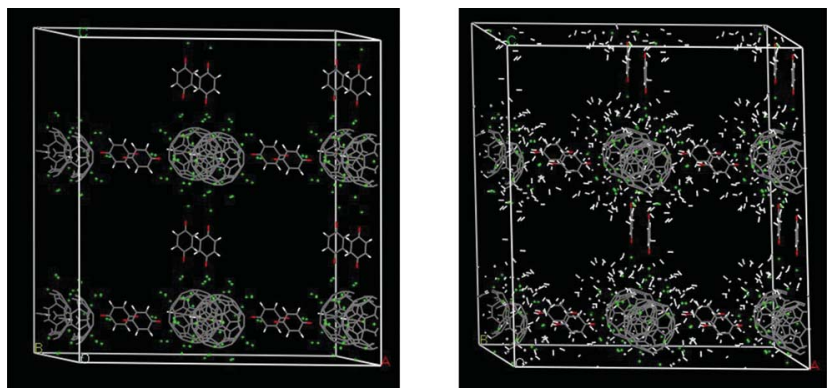

Fig. 5 Optimized structures of $\mathrm{Ca}_{32} \mathrm{C}_{60}$ and $96 \mathrm{H}_{2}-\mathrm{Ca}_{32} \mathrm{C}_{60} 3-$ dimensional framework based on FW-2.

per $\mathrm{H}_{2}$ ). These values are nearly the same as previously reported DFT results of the $\mathrm{H}_{2}$ binding energy on the Li-doped boron carbide nanotubes (GGA-PBE: $0.084 \mathrm{eV}$ per $\mathrm{H}_{2}$; LDA-PWC: $0.196 \mathrm{eV}$ per $\left.\mathrm{H}_{2}\right)^{24}$ and on fullerenes (GGA-PW91: $0.075 \mathrm{eV}$ per $\mathrm{H}_{2}$ ). ${ }^{23}$ Considering that the $\mathrm{H}_{2}$ binding energies on MOF materials are underestimated by GGA-PBE calculations but slightly overestimated by LDA-DFT. ${ }^{21 b, 34,39}$ The binding energies between $\mathrm{H}_{2}$ and FW- 1 framework are likely in the range of $0.10-0.20 \mathrm{eV}$ per $\mathrm{H}_{2}$, which could be further confirmed by the PBE-D results with the binding energy of 0.19-0.29 eV per $\mathrm{H}_{2}$. With full $\mathrm{H}_{2}$ loading, the FW-1 can adsorb $9.2 \mathrm{wt} \% \mathrm{H}_{2}$, satisfying the DOE's criteria.

The hydrogen-storage capacity of $\mathrm{Ca}_{32} \mathrm{C}_{60}$ was reported by Yoon et al. recently. ${ }^{43}$ Their studies suggest that $\mathrm{Ca}$ is superior to

Table 1 Optimized unit length $(\AA)$ of $L i$-coated $\mathrm{C}_{48} \mathrm{~B}_{12}$ fullerene organic framework FW-1 with and without $\mathrm{H}_{2}$ adsorption, and the computed binding (adsorption) energy/ $\mathrm{H}_{2}(\mathrm{eV})$

\begin{tabular}{|c|c|c|c|c|c|}
\hline & \multicolumn{2}{|l|}{ LDA-PWC } & \multicolumn{2}{|l|}{ GGA-PBE } & $\begin{array}{l}\text { PBE-D } \\
\Delta E_{\text {binding }} / \mathrm{H}_{2}(\mathrm{eV})\end{array}$ \\
\hline $14 \mathrm{H}_{2}$ & 17.60 & 0.26 & 17.80 & 0.14 & 0.29 \\
\hline $28 \mathrm{H}_{2}$ & 17.60 & 0.25 & 17.80 & 0.12 & 0.28 \\
\hline $42 \mathrm{H}_{2}$ & 17.55 & 0.23 & 17.80 & 0.10 & 0.23 \\
\hline
\end{tabular}


other metal elements and the hydrogen uptake can be $>8.4 \mathrm{wt} \%$. Here, we use $\mathrm{Ca}_{32} \mathrm{C}_{60}$ clusters to replace metal center of a MOF to construct a 2-D film (Fig. 2c). The $p$-dihydroxybenzene is used as a linker to connect neighbouring $\mathrm{Ca}_{32} \mathrm{C}_{60}$ clusters. The $\mathrm{Ca}-\mathrm{C}$ bond length is in the range of $2.64 \AA-3.27 \AA$ based on LDA-PWC calculations and $2.73 \AA-3.12 \AA$ based on GGA-PBE calculations, respectively. The Hirshfeld charge analysis shows that $\mathrm{Ca}$ atoms carry an average positive charge of $0.19|\mathrm{e}|$. This charge indicates that the adsorption of $\mathrm{H}_{2}$ on $\mathrm{Ca}$ atoms are mainly owing to a polarization mechanism. ${ }^{43}$ The average $\mathrm{Ca}-\mathrm{O}$ bond length is $2.02 \AA$ (LDA-PWC) and 2.05 $\AA$ (GGA-PBE), for which the average binding energy between $p$-dihydroxybenzene linker and $\mathrm{Ca}_{32} \mathrm{C}_{60}$ is $2.73 \mathrm{eV}$ and $2.40 \mathrm{eV}$, respectively. Such a strong binding suggests the network structure of FW-2 is stable. With total 96 hydrogen molecules $\left(3 \mathrm{H}_{2}\right.$ molecules/Ca) loaded into the supercell, the computed average hydrogen binding energy is $0.57 \mathrm{eV}$ per $\mathrm{H}_{2}$ and $0.25 \mathrm{eV}$ per $\mathrm{H}_{2}$, respectively, based on LDAPWC and GGA-PBE calculations. Similarly, the PBE-D calculation gives the binding energy of $0.31 \mathrm{eV}$ per $\mathrm{H}_{2}$. These hydrogen binding-energy values are consistent with previous results, indicating favourable $\mathrm{H}_{2}$ adsorption in the $\mathrm{FW}-2$ system. At the maximum loading (Fig. 4), the $\mathrm{H}_{2}$ gravimetric density is $>8.0 \mathrm{wt}$ $\%$, meeting the DOE 2010 target.

In addition to the layered structures based on FW-2 system, we also design a 3-dimensional network system based on FW-2 (shown in Fig. 5). The PBE-D calculation indicates the average hydrogen binding energy is $0.35 \mathrm{eV}$ per $\mathrm{H}_{2}\left(96 \mathrm{H}_{2}\right.$ per $\left.\mathrm{Ca}_{32} \mathrm{C}_{60}\right)$, close to that for the FW-2 system. The 3-D network is expected to provide a more suitable medium for hydrogen-storage applications.

\section{Conclusion}

In conclusion, two microporous frameworks consisting of organic linkers and exohedral metallofullerene nodes are designed as potential hydrogen storage materials. Both frameworks are predicted to be mechanically stable based on DFT computations. Importantly, both frameworks can store $\mathrm{H}_{2}$ with a gravimetric density up to 8-9.2 $\mathrm{wt} \%$ and with the hydrogen binding energy of $0.15-0.25 \mathrm{eV}$ per $\mathrm{H}_{2}$ and $0.30-0.50 \mathrm{eV}$ per $\mathrm{H}_{2}$, respectively. These energy values lie within the optimal range of $\mathrm{H}_{2}$ adsorption energy for hydrogen storage. The weight percentages also meet the DOE target of 2010 or 2015. The fundamental chemical mechanism underlying the framework design may be exploited for ultimate realization of an ideal hydrogen storage material for automobile application.

\section{Acknowledgements}

YG is supported by a startup fund from Shanghai Institute of Applied Physics, Chinese Academy of Sciences (Y290011011), National Natural Science Foundation of China (21273268) and "Hundred People Project" from Chinese Academy of Sciences. XJW is supported by the National Basic Research Programs of China (no. 2011CB921400, 2012CB 922001), NSFC (Grant no. 21121003, 11004180, 51172223), Hundred People Project of CAS. XCZ is supported by ARL (Grant no. W911NF1020099), NSF
(Grant no. DMR-0820521), and a grant from USTC for (1000plan) Qianren-B summer research. The computational resources utilized in this research were provided by Shanghai Supercomputer Centre, Supercomputing Centre of Chinese Academy of Sciences in Beijing, and University of Nebraska Holland Computing Center.

\section{Notes and references}

1 L. Schlapbach and A. Züttel, Nature, 2001, 414, 353.

2 US Department of Energy, Basic research needs for the hydrogen economy, 2004, p. 32.

3 G. A. Sandrock, J. Alloys Compd., 1999, 293, 877.

4 B. Bogdanovic and G. Sandrock, MRS Bull., 2002, 27, 712.

5 V. P. Balema, K. W. Dennis and V. K. Pecharsky, Chem. Commun., 2000, 1655.

6 P. Chen, Z. Xiong, J. Luo, J. Lin and K. L. Tan, Nature, 2002, 420, 302.

7 H. Lee, J.-W. Lee, D. Y. Kim, J. Park, Y.-T. Seo, H. Zeng, I. L. Moudrakovski, C. I. Ratcliffe and J. A. Ripmeester, Nature, 2005, 434, 743.

8 J. Alper, Science, 2003, 299, 1686.

9 P. Chen, X. Wu, J. Lin and K. L. Tan, Science, 1999, 285, 91. 10 N. L. Rosi, J. Eckert, M. Eddaoudi, D. T. Vodak, J. Kim, M. O'Keeffe and O. M. Yaghi, Science, 2003, 300, 1127.

11 M. Eddaoudi, J. Kim, N. Rosi, D. Vodak, J. Wachter, M. O'Keeffe and O. M. Yaghi, Science, 2002, 295, 469.

12 R. W. P. Wagemans, J. H. van Lenthe, P. E. de Jongh, A. J. van Dillen and K. P. de Jong, J. Am. Chem. Soc., 2005, 127, 16675.

13 (a) P. J. Dyson and J. S. Mclndoe, Angew. Chem., Int. Ed., 2005, 44, 5772; (b) S. K. Brayshaw, M. J. Ingleson, J. C. Green, P. R. Raithby, G. Kociok-Kohn, J. S. Mclndoe and A. S. Weller, Angew. Chem., Int. Ed., 2005, 44, 6875.

14 A. B. Philips and B. S. Shivaram, Phys. Rev. Lett., 2008, 100, 105505.

15 S. Meng, E. Kaxiras and Z. Zhang, Nano Lett., 2007, 7, 663.

16 L. J. Murray, M. Dincă and J. R. Long, Chem. Soc. Rev., 2009, 38, 1294.

17 G. G. Tibbetts, G. P. Meisner and C. H. Olk, Carbon, 2001, 39, 2291.

18 M. Shiraishi, T. Takenobu and M. Ata, Chem. Phys. Lett., 2003, 367, 633.

19 H. Kajiura, S. Tsutsui, K. Kadono, M. Kakuta, M. Ata and Y. Murakami, Appl. Phys. Lett., 2003, 82, 1105.

20 A. C. Dillon, K. M. Jones, T. A. Bekkedahl, C. H. Kiang, D. S. Bethune and M. J. Heben, Nature, 1997, 386, 377.

21 (a) Y. F. Zhao, Y.-H. Kim, A. C. Dillon, M. J. Heben and S. B. Zhang, Phys. Rev. Lett., 2005, 94, 155504; (b) Y.-H. Kim, Y. Zhao, A. Williamson, M. Heben and S. B. Zhang, Phys. Rev. Lett., 2006, 96, 016102.

22 Q. Sun, Q. Wang, P. Jena and Y. Kawazoe, J. Am. Chem. Soc., 2005, 127, 14582.

23 Q. Sun, P. Jena, Q. Wang and M. Marquez, J. Am. Chem. Soc., 2006, 128, 9741.

24 (a) X. Wu, Y. Gao and X. C. Zeng, J. Phys. Chem. C, 2008, 112, 8458; (b) M. Wu, Y. Gao, Z. Y. Zhang and X. C. Zeng, Nanoscale, 2012, 4, 915. 
25 (a) Q. Sun, Q. Wang and P. Jena, Appl. Phys. Lett., 2009, 94, 013111; (b) M. Yoon, S. Yang, C. Hicke, E. Wang, D. Geohegan and Z. Zhang, Phys. Rev. Lett., 2008, 100, 206806.

26 Q. Wang, Q. Sun, P. Jena and Y. Kawazoe, Theoretical Study of Hydrogen Storage in Ca-Coated Fullerenes, J. Chem. Theory Comput., 2009, 5, 374.

27 J. L. C. Rowsell, A. R. Millward, K. S. Park and O. M. Yaghi, J. Am. Chem. Soc., 2004, 126, 5666.

28 A. G. Wong-Foy, A. J. Matzger and O. M. Yaghi, J. Am. Chem. Soc., 2006, 128, 3494.

29 M. Dincă, A. Dailly, Y. Liu, C. M. Brown, D. A. Neumann and J. R. Long, J. Am. Chem. Soc., 2006, 128, 16876.

30 H. M. El-Kaderi, J. R. Hunt, J. L. Mendoza-Cortés, A. P. Côté, R. E. Taylor, M. O'Keeffe and O. M. Yaghi, Science, 2007, 316, 268. 31 S. H. Yang, X. Lin, A. J. Blake, G. S. Walker, P. Hubberstey, N. R. Champness and M. Schroder, Nat. Chem., 2009, 1, 487. 32 H. Furukawa, N. Ko, Y. B. Go, N. Aratani, S. B. Choi, E. Choi, A. Ö. Yazaydin, R. Q. Snurr, M. O'Keeffe, J. Kim and O. M. Yaghi, Science, 2010, 329, 424.

33 H. Deng, S. Grunder, K. E. Cordova, C. Valente, H. Furukawa, M. Hmadeh, F. Gándara, A. C. Whalley, Z. Liu, S. Asahina,
H. Kazumori, M. O'Keeffe, O. Terasaki, J. F. Stoddart and O. M. Yaghi, Science, 2012, 336, 1018.

34 J. Bacsa, R. J. Less, H. E. Skelton, Z. Soracevic, A. Steiner, T. C. Wilson, P. T. Wood and D. S. Wright, Angew. Chem., Int. Ed., 2011, 50, 8279.

35 D. Rao, R. Lu, Z. Meng, G. Xu, E. Kan, Y. Liu, C. Xiao and K. Deng, Mol. Simul., 2013, 39, 968.

36 S. S. Han and W. A. Goddard III, J. Am. Chem. Soc., 2007, 129, 8422-8423.

37 J. P. Perdew and Y. Wang, Phys. Rev. B: Condens. Matter, 1992, 45, 13244.

38 J. P. Perdew, J. Burke and M. Ernzerhof, Phys. Rev. Lett., 1996, 77, 3865.

39 Y. Gao and X. C. Zeng, J. Phys.: Condens. Matter, 2007, 19, 386220.

40 S. Grimme, J. Comput. Chem., 2006, 27, 1787.

41 (a) B. Delley, J. Chem. Phys., 1990, 92, 508; (b) B. Delley, J. Chem. Phys., 2000, 113, 7756.

42 B. K. Rao and P. Jena, Europhys. Lett., 1992, 20, 307.

43 M. Yoon, S. Y. Yang, C. Hicke, E. Wang, D. Geohagan and Z. Y. Zhang, Phys. Rev. Lett., 2008, 100, 206806. 